Research Article

\title{
Investigation of a Spin-0 Massive Charged Particle Subject to a Homogeneous Magnetic Field with Potentials in a Topologically Trivial Flat Class of Gödel-Type Space-Time
}

\author{
Faizuddin Ahmed iD \\ Ajmal College of Arts and Science, Dhubri, 783324 Assam, India \\ Correspondence should be addressed to Faizuddin Ahmed; faizuddinahmed15@gmail.com
}

Received 24 February 2020; Accepted 22 May 2020; Published 10 June 2020

Academic Editor: Grégory Moreau

Copyright (c) 2020 Faizuddin Ahmed. This is an open access article distributed under the Creative Commons Attribution License, which permits unrestricted use, distribution, and reproduction in any medium, provided the original work is properly cited.

In this paper, we investigate the relativistic quantum dynamics of spin-0 massive charged particle subject to a homogeneous magnetic field in the Gödel-type space-time with potentials. We solve the Klein-Gordon equation subject to a homogeneous magnetic field in a topologically trivial flat class of Gödel-type space-time in the presence of Cornell-type scalar and Coulombtype vector potentials and analyze the effects on the energy eigenvalues and eigenfunctions.

\section{Introduction}

The first solution to Einstein's field equations containing closed time-like curves is the cylindrical symmetry Gödel rotating universe [1]. Reboucas et al. [2-4] investigated the Gödel-type solutions characterized by vorticity, which represents a generalization of the original Gödel metric with possible sources, and analyzed the problem of causality. The line element of Gödel-type solution is given by

$$
d s^{2}=-\left(d t+A_{i} d x^{i}\right)^{2}+\delta_{i j} d x^{i} d x^{j}
$$

where the spatial coordinates of the space-time are represented by $x^{i}$ and $i, j=1,2,3$. The different classes of Gödeltype solutions have been discussed in [5].

Investigation of relativistic quantum dynamics of spinzero and spinhalf particles in the Gödel universe and Gödel-type space-times as well as the Schwarzschild and the Kerr black hole solution has been addressed by several authors. The study of relativistic wave equations, particularly the Klein-Gordon and Dirac equations, in the background of Gödel-type space-time was first conducted in [6]. The close relation between the relativistic energy levels of a scalar par- ticle in the Som-Raychaudhuri space-time with the Landau levels was studied in [7]. The same problem in the SomRaychaudhuri space-time was investigated and compared with the Landau levels [8]. The Klein-Gordon equation in the background of Gödel-type space-times with a cosmic string was studied in [9], and the similarity of the energy levels with Landau levels in flat space was analyzed. The Klein-Gordon oscillator in the background of Gödel-type space-time under the influence of topological defects was studied in [10]. The relativistic quantum dynamics of a scalar particle in the presence of external fields in the SomRaychaudhuri space-time under the influence of topological defects was studied in [11]. The relativistic quantum motion of spin-0 particles in a flat class of Gödel-type space-time was studied in [12]. The study of the spin-0 system of the DKP equation in a flat class of Gödel-type space-time was studied in [13]. In all the above systems, the influence of topological defects and vorticity parameter characterizing the space-time on the relativistic energy eigenvalues was analyzed. Linear confinement of a scalar particle in the Som-Raychaudhuri space-time with a cosmic string was studied in [14] (see also [15]). The behavior of a scalar particle with Yukawa-like confining potential in the Som-Raychaudhuri space-time in the presence of topological defects was investigated in [16]. The 
ground state of a bosonic massive charged particle in the presence of external fields in Gödel-type space-time was investigated in [17] (see also [18]). The relativistic quantum dynamics of spin-zero particles in 4D curved space-time with the cosmic string subject to a homogeneous magnetic field was studied in [19]. In addition, the relativistic wave equations in the $(1+2)$-dimensional rotational symmetry spacetime background were investigated in [20-25].

Furthermore, Dirac and Weyl fermions in the background of the Som-Raychaudhuri space-times in the presence of topological defects with torsion were studied in [26]. Weyl fermions in the background of the Som-Raychaudhuri space-times in the presence of topological defects were studied in [27] (see Refs. [28, 29]). The relativistic wave equations for spinhalf particles in the Melvin space-time, a kind of spacetime where the metric is determined by a magnetic field, were studied in [30]. The Fermi field and Dirac oscillator in the Som-Raychaudhuri space-time were studied in [31]. The Fermi field with scalar and vector potentials in the SomRaychaudhuri space-time was investigated in [32]. The Dirac particles in a flat class of Gödel-type space-time were studied in [5]. Dirac fermions in the $(1+2)$-dimensional rotational symmetry space-time background were investigated in [33].

The relativistic quantum dynamics of a scalar particle subject to different confining potentials has been studied in several areas of physics by various authors. The relativistic quantum dynamics of scalar particles subject to Coulombtype potential was investigated in [34-37]. It is worth mentioning studies that have dealt with Coulomb-type potential in the propagation of gravitational waves [38], quark models [39], and relativistic quantum mechanics [40-43]. Linear confinement of scalar particles in a flat class of Gödel-type space-time was studied in [44]. The Klein-Gordon equation with vector and scalar potentials of Coulomb type under the influence of noninertial effect in cosmic string spacetime was studied in [45]. The Klein-Gordon oscillator in the presence of Coulomb-type potential in the background space-time generated by a cosmic string was studied in [43, 46]. Other works on the relativistic quantum dynamics are the Klein-Gordon scalar field subject to a Cornell-type potential [47] and a survey on the Klein-Gordon equation in Gödel-type space-time [48].

Our aim in this paper is to investigate the quantum effects on a bosonic massive charged particle by solving the KleinGordon equation subject to a homogeneous magnetic field in the presence of Cornell-type scalar and Coulomb-type vector potentials in Gödel-type space-time. We see that the presence of a magnetic field as well as various potentials modifies the energy spectrum.

\section{Bosonic Charged Particle: The KG Equation}

The relativistic quantum dynamics of a charged particle of modifying mass $m \longrightarrow m+S$, where $S$ is the scalar potential which is described by the following equation [42]:

$$
\left[\frac{1}{\sqrt{-g}} D_{\mu}\left(\sqrt{-g} g^{\mu v} D_{v}\right)-(m+S)^{2}-\xi R\right] \Psi=0,
$$

where $g$ is the determinant of a metric tensor with $g^{\mu \nu}$ as its inverse, $D_{\mu}=\partial_{\mu}-i e A_{\mu}$ is the minimal substitution, in which $e$ is the electric charge and $A_{\mu}$ is the electromagnetic fourvector potential, and $\xi$ is the nonminimal coupling constant with the background curvature.

We choose the electromagnetic four-vector potential $A_{\mu}=\left(-V, A^{\sim}\right)$ with [49]

$$
\begin{aligned}
A_{y} & =-x B_{0}, \\
\vec{A} & =\left(0, A_{y}, 0\right),
\end{aligned}
$$

such that the constant magnetic field is along the axis $\vec{B}=$ $\vec{\nabla} \times \vec{A}=-B_{0} \widehat{z}$.

Consider the following stationary space-time [50] (see $[5,12,13,44,51])$ in the Cartesian coordinates $\left(x^{0}=t\right.$, $\left.x^{1}=x, x^{2}=y, x^{3}=z\right)$ which is given by

$$
d s^{2}=-\left(d t+\alpha_{0} x d y\right)^{2}+\delta_{i j} d x^{i} d x^{j}
$$

where $\alpha_{0}>0$ is a real positive constant. In Ref. [5], we have discussed different classes of Gödel-type space-time. For the space-time geometry (4), it belongs to a linear or flat class of Gödel-type metrics. The parameter $\alpha_{0}=2 \Omega$, where $\Omega$ characterizes the vorticity parameter of the space-time. For $\Omega \longrightarrow 0$, the study space-time reduces to four-dimensional flat Minkowski metric.

The determinant of the corresponding metric tensor $g_{\mu \nu}$ is

$$
\operatorname{det} g=-1 \text {. }
$$

The scalar curvature of the metric is

$$
R=\frac{\alpha_{0}^{2}}{2}=2 \Omega^{2}
$$

For the space-time geometry (4), equation (2) becomes

$$
\begin{aligned}
& {\left[-\left(\frac{\partial}{\partial t}+i e V\right)^{2}+\left\{\left(\frac{\partial}{\partial y}-i e A_{y}\right)-2 \Omega x\left(\frac{\partial}{\partial t}+i e V\right)\right\}^{2}\right.} \\
& \left.+\frac{\partial^{2}}{\partial x^{2}}+\frac{\partial^{2}}{\partial z^{2}}-(m=S)^{2}-2 \xi \Omega^{2}\right] \Psi=0 .
\end{aligned}
$$

Since the metric is independent of $t, y, z$, one can choose the following ansatz for the function $\Psi$ :

$$
\Psi(t, x, y, z)=e^{i(-E t+l y+k z)} \psi(x)
$$

where $E$ is the total energy of the particle, $l=0, \pm 1, \pm 2, \cdots$ are the eigenvalues of the $y$-component operator, and $-\infty<k<$ $\infty$ is the eigenvalues of the $z$-component operator. 
Substituting the ansatz (equation (8)) into equation (7), we obtain the following differential equation for $\psi(x)$ :

$$
\begin{aligned}
& {\left[\frac{d^{2}}{d x^{2}}+(E-e V)^{2}-k^{2}-\left\{\left(l+e B_{0} x\right)+2 \Omega x(E-e V)\right\}^{2}\right.} \\
& \left.-(m+S)^{2}-2 \xi \Omega^{2}\right] \psi(x)=0 .
\end{aligned}
$$

2.1. Interaction with Cornell-Type and Coulomb-Type Potentials. Here, we study a spin-0 massive charged particle by solving the Klein-Gordon equation in the presence of external fields in a flat class of Gödel-type space-time subject to Cornell-type scalar and Coulomb-type vector potentials. We obtain the energy eigenvalues and eigenfunctions and analyze the effects due to various physical parameters.

The Cornell-type potential contains a confining (linear) term besides the Coulomb interaction and has been successfully accounted for the particle physics data [52]. This type of potential is a particular case of the quark-antiquark interaction, which has one more harmonic-type term [53]. The Coulomb potential is responsible for the interaction at small distances, and the linear potential leads to the confinement. The quark-antiquark interaction potential has been studied in the ground state of three quarks [54] and systems of bound heavy quarks [55-57]. This type of interaction has been studied by several authors $([11,23,42,58-64])$.

We consider the scalar $S$ to be Cornell-type [42].

$$
S=\frac{\eta_{c}}{x}+\eta_{L} x
$$

where $\eta_{c}$ and $\eta_{L}$ are the Coulombic and confining potential constants, respectively.

Another potential that we are interested in here is the Coulomb-type potential which we discussed in Introduction. Therefore, the Coulomb-type vector potential is given by

$$
V=\frac{\xi_{c}}{x}
$$

where $\xi_{c}$ is the Coulombic potential constants.

Substituting the potentials (equations (10) and (11)) into equation (9), we obtain the following equation:

$$
\left[\frac{d^{2}}{d x^{2}}+\lambda-\omega^{2} x^{2}-\frac{j^{2}}{x^{2}}-\frac{a}{x}-b x\right] \psi(x)=0,
$$

where we have defined

$$
\begin{aligned}
\lambda & =E^{2}-m^{2}-k^{2}-2 \eta_{c} \eta_{L}-2 \xi \Omega^{2}-\left(l-2 e \Omega \xi_{c}\right)^{2}, \\
\omega & =\sqrt{4\left(\Omega E+m \omega_{c}\right)^{2}+\eta_{L}^{2}} \\
j & =\sqrt{\eta_{c}^{2}-e^{2} \xi_{c}^{2}} \\
a & =2\left(e \xi_{c} E+m \eta_{c}\right) \\
b & =2\left[m \eta_{L}+\omega\left(l-2 e \Omega \xi_{c}\right)\right] \\
\omega_{c} & =\frac{e B_{0}}{2 m}
\end{aligned}
$$

is called the cyclotron frequency of the particle moving in the magnetic field. Let us define a new variable $r=\sqrt{ } \omega x$, equation (12) becomes

$$
\left[\frac{d^{2}}{d r^{2}}+\beta-r^{2}-\frac{j^{2}}{r^{2}}-\frac{\eta}{r}-\theta r\right] \psi(r)=0,
$$

where

$$
\begin{aligned}
& \beta=\frac{\lambda}{\omega}, \\
& \eta=\frac{a}{\sqrt{\omega}}, \\
& \theta=\frac{b}{\omega^{3 / 2}} .
\end{aligned}
$$

We now use the appropriate boundary conditions to investigate the bound state solution in this problem. It is known in relativistic quantum mechanics that the radial wave functions must be regular at both $r \longrightarrow 0$ and $r \longrightarrow$ $\infty$. Then, we proceed with the analysis of the asymptotic behavior of the radial eigenfunctions at origin and in the infinite. These conditions are necessary since the wave functions must be well behaved in these limits, and thus, the bound states of energy eigenvalues for this system can be obtained. Suppose the possible solution to equation (14) is

$$
\psi(r)=r^{j} e^{-(1 / 2)(\theta+r) r} H(r) .
$$

Substituting the solution (equation (16)) into equation (14), we obtain

$$
\frac{d^{2} H}{d r^{2}}+\left[\frac{\gamma}{r}-\theta-2 r\right] \frac{d H}{d r}+\left[-\frac{\chi}{r}+\Theta\right] H(r)=0,
$$

where

$$
\begin{aligned}
& \gamma=1+2 j, \\
& \Theta=\beta+\frac{\theta^{2}}{4}-2(1+j), \\
& \chi=\eta+\frac{\theta}{2}(1+2 j) .
\end{aligned}
$$


Equation (17) is the biconfluent Heun's differential equation $[42,43,46,59-63,65,66]$ with $H(r)$ as the Heun polynomial function.

Writing the function $H(r)$ as a power series expansion around the origin, we can obtain [49]

$$
H(r)=\sum_{i=0}^{\infty} c_{i} r^{i}
$$

Substituting the series solution into equation (17), we obtain the following recurrence relation:

$$
c_{n+2}=\frac{1}{(n+2)(n+1+\gamma)}\left[\{\chi+\theta(n+1)\} c_{n+1}-(\Theta-2 n) c_{n}\right] \text {. }
$$

Few coefficients of the series solution are

$$
\begin{aligned}
& c_{1}=\left(\frac{\eta}{\gamma}+\frac{\theta}{2}\right) c_{0}, \\
& c_{2}=\frac{1}{2(1+\gamma)}\left[(\chi+\theta) c_{1}-\Theta c_{0}\right] .
\end{aligned}
$$

As the function $H(r)$ has a power series expansion around the origin in equation (19), then the relativistic bound state solution can be achieved by imposing that the power series expansion becomes a polynomial of degree $n$ and we obtain a finite degree polynomial for the biconfluent Heun series. Furthermore, the wave function $\psi$ must vanish at $r \longrightarrow \infty$ for this finite degree polynomial of power series; otherwise, the function diverges for large values of $r$. Therefore, we must truncate the power series expansion $H(r)$ as a polynomial of degree $n$ by imposing the following two conditions $[11,14,42-44,46,59-63,67,68]$ :

$$
\begin{aligned}
\Theta & =2 n(n=1,2, \cdots), \\
c_{n+1} & =0 .
\end{aligned}
$$

By analyzing the condition $\Theta=2 n$, we have the seconddegree eigenvalue equation:

$$
\begin{aligned}
E_{n, l}^{2}= & m^{2}+k^{2}+2 \eta_{c} \eta_{L}+2 \xi \Omega^{2}+2 \omega\left(n+1+\sqrt{\eta_{c}^{2}-e^{2} \xi_{c}^{2}}\right) \\
& -\frac{m^{2} n_{L}^{2}}{\omega^{2}}-\frac{2 m n_{L}\left(l-2 e \xi_{c} \Omega\right)}{\omega} .
\end{aligned}
$$

The corresponding eigenfunctions are given by

$$
\psi_{n, l}(r)=r \sqrt{\eta_{c}^{2}-e^{2} \xi_{c}^{2}} e^{-(1 / 2)(r+\theta) r} H(r) .
$$

Note that equation (23) does not represent the general expression of the eigenvalue problem. One can obtain the individual energy eigenvalues one by one, that is, $E_{1}, E_{2}, E_{3}$, $\cdots$., by imposing the additional recurrence condition $c_{n+1}=$
0 on the eigenvalues. The solution with Heun's equation makes it possible to obtain the individual eigenvalues one by one as done in $[11,14,42-44,46,59-63,67,68]$. In order to analyze the above conditions, we must assign values to $n$. In this case, consider $n=1$, which means we want to construct a first-degree polynomial to $H(r)$. With $n=1$, we have $\Theta=2$ and $c_{2}=0$ which implies equation (21):

$$
\begin{array}{r}
(\chi+\theta) c_{1}=2 c_{0} \Rightarrow \frac{\eta}{\gamma}+\frac{\theta}{2}=\frac{2}{\chi+\theta}, \\
\left(\frac{a_{1, l}}{1+2 j}+\frac{b_{1, l}}{2 \omega_{1, l}}\right)\left(a_{1, l}+\frac{b_{1, l}}{\omega_{1, l}}\left(j+\frac{3}{2}\right)\right)=2 \omega_{1, l}, \\
\omega_{1, l}^{3}-\frac{a^{2}}{2(1+2 j)} \omega_{1, l}^{2}-a b\left(\frac{1+j}{1+2 j}\right) \omega_{1, l}-\frac{b^{2}}{8}(3+2 j)=0,
\end{array}
$$

a constraint on the physical parameter $\omega_{1, l}$. The relation given in equation (25) gives the possible values of the parameter $\omega_{1, l}$ that permit us to construct a first-degree polynomial to $H(r)$ for $n=1[42,43,46,59]$. Note that its values change for each quantum number $n$ and $l$, so we have labeled $\omega \longrightarrow \omega_{n, l}$. In this way, we obtain the following energy eigenvalue $E_{1, l}$ :

$$
\begin{aligned}
E_{1, l}= & \pm\left\{m^{2}+k^{2}+2 \eta_{c} \eta_{L}+2 \xi \Omega^{2}+2\left(2+\sqrt{\eta_{c}^{2}-e^{2} \xi_{c}^{2}}\right) \omega_{1, l}\right. \\
& \left.-\frac{m^{2} \eta_{L}^{2}}{\omega_{1, l}^{2}}-\frac{2 m \eta_{L}\left(l-2 e \xi_{c} \Omega\right)}{\omega_{1, l}}\right\} .
\end{aligned}
$$

Then, by substituting the real solution $\omega_{1, l}$ from equation (25) into equation (26), it is possible to obtain the allowed values of the relativistic energy levels for the radial mode $n=1$ of a position-dependent mass system. We can see that the lowest energy state is defined by the real solution of the algebraic equation (equation (25)) plus the expression given in equation (26) for the radial mode $n=1$, instead of $n=0$. This effect arises due to the presence of Cornell-type potential in the system. Note that it is necessary physically that the lowest energy state is $n=1$ and not $n=0$; otherwise, the opposite would imply that $c_{1}=0$ which is not possible.

The corresponding radial wave function for $n=1$ is given by

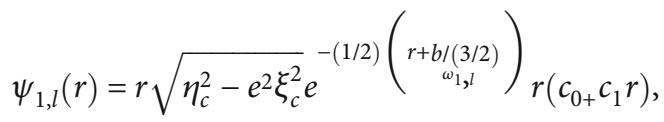

where

$$
c_{1}=\frac{1}{\sqrt{\omega_{1, l}}}\left[\frac{a}{1+2 \sqrt{\eta_{c}^{2}-e^{2} \xi_{c}^{2}}}+\frac{b}{2 \omega_{1, l}}\right] c_{0} .
$$


2.2. Interaction without Potential. Here, we study a spin-0 massive charged particle by solving the Klein-Gordon equation in the presence of external fields in Gödel-type space-time without potential and obtain the relativistic energy eigenvalue.

We choose here zero scalar and vector potentials, $S=$ $0=V$. In that case, equation (9) becomes

$$
\left[\frac{d^{2}}{d x^{2}}+E^{2}-m^{2}-k^{2}-l^{2}-2 \xi \Omega^{2}-\omega^{2} x^{2}-2 \omega l x\right] \psi(x)=0
$$

The above equation can be expressed as

$$
\left[\frac{d^{2}}{d x^{2}}+E^{2}-m^{2}-k^{2}-2 \xi \Omega^{2}-\omega^{2}\left(x+\frac{l}{\omega}\right)^{2}\right] \psi(x)=0
$$

Let us define a new variable $r=(x+(l / \omega))$; equation (30) becomes

$$
\psi^{\prime \prime}(r)+\left(\delta-\omega^{2} r^{2}\right) \psi(r)=0
$$

where

$$
\delta=E^{2}-m^{2}-k^{2}-2 \xi \Omega^{2} .
$$

Again, introducing a new variable $\rho=\sqrt{ } \omega r$ into equation (31), we obtain

$$
\psi^{\prime \prime}(\rho)+\left(\frac{\delta}{\omega}-\rho^{2}\right) \psi(\rho)=0
$$

which is similar to a harmonic-type oscillator equation. Therefore, the energy eigenvalue equation is

$$
\begin{aligned}
\frac{\delta}{\omega}= & 2 n+1 \Rightarrow \delta=(2 n+1) \omega \Rightarrow E_{n}^{2}-2 \Omega(2 n+1) E_{n} \\
& -m^{2}-k^{2}-2 \xi \Omega^{2}-2 m \omega_{c}(2 n+1)=0 .
\end{aligned}
$$

The energy eigenvalues associated with the $n^{\text {th }}$ modes are

$$
\begin{aligned}
E_{n}= & (2 n+1) \Omega \\
& +\sqrt{(2 n+1)^{2} \Omega^{2}+m^{2}+k^{2}+2 \xi \Omega^{2}+2 m \omega_{c}(2 n+1)} \\
= & (2 n+1) \Omega \\
& +\sqrt{(2 n+1)^{2} \Omega^{2}+m^{2}+k^{2}+2 \xi \Omega^{2}+\left|e B_{0}\right|(2 n+1)},
\end{aligned}
$$

where $n=0,1,2, \cdots$. We can see that the energy eigenvalues (35) depend on the parameter $\Omega$ characterizing the vorticity parameter of the space-time geometry and the external magnetic field $B_{0}$ as well as the nonminimal coupling constant $\xi$ with the background curvature.
In the absence of external magnetic fields, $B_{0} \longrightarrow 0$, and without the nonminimal coupling constant, $\xi \longrightarrow 0$, the eigenvalue (35) becomes

$$
E_{n}=(2 n+1) \Omega+\sqrt{(2 n+1)^{2} \Omega^{2}+m^{2}+k^{2}} .
$$

Equation (36) is the energy eigenvalues of the spin-0 particle in the background of a flat class of Gödel-type spacetime and consistent with the result in [12]. Thus, we can see that the energy eigenvalues (35) in comparison to the result in [12] get modified due to the presence of external fields and the nonminimal coupling constant with the background curvature.

Therefore, the individual energy levels for $n=0,1$ using (35) are follows:

$$
\begin{aligned}
& n=0: E_{0}=\Omega+\sqrt{\Omega^{2}+2 \xi \Omega^{2}+2 m \omega_{c}+m^{2}+k^{2}}, \\
& n=1: E_{1}=3 \Omega+\sqrt{9 \Omega^{2}+2 \xi \Omega^{2}+6 m \omega_{c}+m^{2}+k^{2}} .
\end{aligned}
$$

A special case corresponds to $m=0=k$, and the energy eigenvalues (35) reduce to

$$
E_{n}=(2 n+1) \Omega+\sqrt{(2 n+1)^{2} \Omega^{2}+2 \xi \Omega^{2}+\left|e B_{0}\right|(2 n+1)} .
$$
follows:

The individual energy levels for $n=0,1$ in that case are as

$$
\begin{aligned}
& n=0: E_{0}=\Omega+\sqrt{\Omega^{2}+2 \xi \Omega^{2}+\left|e B_{0}\right|} \\
& n=1: E_{1}=3 \Omega+\sqrt{9 \Omega^{2}+2 \xi \Omega^{2}+3\left|e B_{0}\right|} .
\end{aligned}
$$

And others are in the same way. We can see that the presence of the external magnetic field $B_{0}$ as well as the nonminimal coupling constant $\xi$ causes asymmetry in the energy levels, and hence, the energy levels are not equally spaced.

The eigenfunctions are given by

$$
\psi_{n}(\rho)=|N| H_{n}(\rho) e^{-\left(\rho^{2} / 2\right)},
$$

where $|N|=\sqrt{1 / 2^{n} n ! \sqrt{\pi}}$ is the normalization constant and $H_{n}(\rho)$ are the Hermite polynomials and are defined as

$$
\begin{aligned}
H_{n}(\rho) & =(-1)^{n} e^{\rho^{2}} \frac{d^{n}}{d p^{n}}\left(e^{-\rho^{2}}\right), \\
\int_{-\infty}^{\infty} e^{-\rho^{2}} H_{n}(\rho) H_{m}(\rho) d \rho & =\sqrt{\pi} 2^{n} n ! \delta_{n m} .
\end{aligned}
$$

\section{The Klein-Gordon Oscillator}

Here, we study a spin-0 massive charged particle by solving the Klein-Gordon equation of the Klein-Gordon oscillator in the presence of external fields in Gödel-type space-time 
subject to Cornell-type scalar and Coulomb-type vector potentials. We analyze the effects on the relativistic energy eigenvalue and corresponding eigenfunctions due to various physical parameters.

To couple the Klein-Gordon field with the oscillator [69, 70], we adopted the generalization of Mirza and Mohadesi's prescription [71], in which the following change in the momentum operator is considered [71]:

$$
p_{\mu} \longrightarrow p_{\mu}+i m \omega_{0} X_{\mu}
$$

where $m$ is the particle mass at rest, $\omega_{0}$ is the frequency of the oscillator, and $X_{\mu}=(0, x, 0,0)$, with $x$ being the distance of the particle. In this way, the Klein-Gordon oscillator equation becomes

$$
\frac{1}{\sqrt{-g}}\left(D_{\mu}+m \omega_{0} X_{\mu}\right) \sqrt{-g} g^{\mu v}\left(D_{v}+m \omega_{0} X_{v}\right) \Psi=(m+S)^{2} \Psi .
$$

Using the space-time (2), we obtain the following equation:

$$
\begin{aligned}
& {\left[-\left(\frac{\partial}{\partial t}+i e V\right)^{2}+\left\{\left(\frac{\partial}{\partial y} i e A_{y}\right)-\alpha x\left(\frac{\partial}{\partial t}+i e V\right)\right\}^{2}\right.} \\
& \left.\quad+\frac{\partial}{\partial z^{2}}+\left(\frac{\partial}{\partial x}+m \omega_{0} x\right)\left(\frac{\partial}{\partial x}-m \omega_{0} x\right)-(m+S)^{2}\right] \psi=0 .
\end{aligned}
$$

Using the ansatz (8) into equation (44), we arrive at the following equation:

$$
\begin{gathered}
\frac{d^{2} \psi}{d x^{2}}+\left[(E-e V)^{2}-\left\{\alpha_{0} x(E-e V)+\left(l-e A_{y}\right)\right\}^{2}\right. \\
\left.-k^{2}-m \omega_{0}-m^{2} \omega_{0}^{2} x^{2}-(m+S)^{2}\right] \psi=0 .
\end{gathered}
$$

Substituting the potentials (equations (3), (10), and (11)) into equation (45), we obtain the following equation:

$$
\psi^{\prime \prime}(x)+\left[\tilde{\lambda}-\tilde{\omega}^{2} x^{2}-\frac{j^{2}}{x^{2}}-\frac{a}{x}-\tilde{b} x\right] \psi(x)=0,
$$

where we have defined

$$
\begin{aligned}
\tilde{\lambda}-E^{2}-m^{2} & -k^{2}-2 \eta_{c} \eta_{L}-2 \xi \Omega^{2}-\left(l-2 e \Omega \xi_{c}\right)^{2}-m \omega_{0}, \\
\tilde{\omega} & =\sqrt{4\left(\Omega E+m \omega_{c}\right)^{2}+m^{2} \omega_{0}^{2}+\eta_{L}^{2}} \\
j & =\sqrt{\eta_{c}^{2}-e^{2} \xi_{c}^{2}}, \\
a & =2\left(e \xi_{c} E+m \eta_{c}\right) \\
\tilde{b} & =2\left[m \eta_{L}+\tilde{\omega}\left(l-2 e \Omega \xi_{c}\right)\right] .
\end{aligned}
$$

Let us define a new variable $r=\sqrt{\tilde{\omega}} x$, equation (46) becomes

$$
\left[\frac{d^{2}}{d r^{2}}+\tilde{\beta}-r^{2}-\frac{j^{2}}{r^{2}}-\frac{\tilde{\eta}}{r}-\tilde{\theta} r\right] \psi(r)=0,
$$

where

$$
\begin{aligned}
& \tilde{\beta}=\frac{\lambda}{\tilde{\omega}}, \\
& \tilde{\eta}=\frac{a}{\sqrt{\tilde{\omega}}}, \\
& \tilde{\theta}=\frac{\tilde{b}}{\tilde{\omega}^{3 / 2}} .
\end{aligned}
$$

Suppose the possible solution to equation (48) is

$$
\psi(r)=r^{j} e^{-(1 / 2)(\theta+r) r} H(r) .
$$

Substituting the solution (equation (50)) into equation (48), we obtain

$$
H^{\prime \prime}(r)+\left[\frac{\gamma}{r}-\tilde{\theta}-2 r\right] H^{\prime}(r)+\left[-\frac{\tilde{\chi}}{r}+\Theta\right] H(r)=0,
$$

where $\gamma$ is given earlier and

$$
\begin{aligned}
& \tilde{\Theta}=\tilde{\beta}+\frac{\tilde{\theta}^{2}}{4}-2(1+j), \\
& \tilde{\chi}=\tilde{\eta}+\frac{\tilde{\theta}}{2}(1+2 j) .
\end{aligned}
$$

Equation (51) is the biconfluent Heun's differential equation $[42,43,46,59-63,65,66]$.

Substituting the series solution (equation (19)) into equation (51), we obtain the following recurrence relation:

$$
c_{n+2}=\frac{1}{(n+2)(n+1+\gamma)}\left[\{\tilde{\chi}+\tilde{\theta}(n+1)\} c_{n+1}-(\tilde{\Theta}-2 n) c_{n}\right] .
$$

Few coefficients of the series solution are

$$
\begin{aligned}
& c_{1}=\left(\frac{\tilde{\eta}}{\gamma}+\frac{\tilde{\theta}}{2}\right) c_{0}, \\
& c_{2}=\frac{1}{2(1+\gamma)}\left[(\tilde{\chi}+\tilde{\theta}) c_{1}-\Theta c_{0}\right] .
\end{aligned}
$$

The power series expansion becomes a polynomial of degree $n$ by imposing the following two conditions [11, 14, $42-44,46,59-63,67,68]$ : 


$$
\begin{aligned}
\tilde{\Theta} & =2 n(n=1,2, \cdots), \\
c_{n+1} & =0 .
\end{aligned}
$$

Using the first condition, we obtain the following energy eigenvalues:

$$
\begin{aligned}
E_{n, l}^{2}= & m^{2}+k^{2} 2 \eta_{c} \eta_{L}+2 \xi \Omega^{2}+m \omega_{0}+2\left(n+1+\sqrt{\eta_{c}^{2} \xi_{c}^{2}}\right) \tilde{\omega} \\
& -\frac{m^{2} \eta_{L}^{2}}{\tilde{\omega}^{2}}-\frac{2 m \eta_{L}\left(l-2 e \xi_{c} \Omega\right)}{\tilde{\omega}} .
\end{aligned}
$$

The corresponding eigenfunctions are given by

$$
\psi_{n, l}(r)=r \sqrt{\eta_{c}^{2}-e^{2} \xi_{c}^{2}} e^{-(1 / 2)(r+\tilde{0}) r} H(r) .
$$

As done earlier, we obtain the individual energy levels by imposing the recurrence condition $c_{n+1}=0$. For $n=1$, we have $c_{2}=0$ which implies from equation (54).

$$
\tilde{\omega}_{1, l}^{3}-\frac{a^{2}}{2(1+2 j)} \tilde{\omega}_{1, l}^{2}-a \tilde{b}\left(\frac{1+j}{1+2 j}\right) \tilde{\omega}_{1, l}-\frac{\tilde{b}^{2}}{8}(3+2 j)=0,
$$

a constraint on the physical parameter $\sim \omega_{1, l}$. The relation given in equation (58) gives the possible values of the parameter $\sim \omega_{1, l}$ that permit us to construct a first-degree polynomial to $H(r)$ for $n=1[42,43,46,59]$. In this way, we obtain the following second-degree algebraic equation for $E_{1, l}$ :

$$
\begin{aligned}
E_{1, l}= & \pm\left\{m^{2}+k^{2}+2 \eta_{c} \eta_{L}+2 \xi \Omega^{2}+2\left(2+\sqrt{\eta_{c}^{2}-e^{2} \xi_{c}^{2}}\right) \tilde{\omega}_{1, l}\right. \\
& \left.-\frac{m^{2} n_{L}^{2}}{\tilde{\omega}_{1, l}^{2}}-\frac{2 m \eta_{L}\left(l-2 e \xi_{c} \Omega\right)}{\tilde{\omega}_{1, l}}\right\}^{1 / 2} .
\end{aligned}
$$

Then, by substituting the real solution $\sim \omega_{1, l}$ from equation (58) into equation (59), it is possible to obtain the allowed values of the relativistic energy levels for the radial mode $n=1$ of a position-dependent mass system. We can see that the lowest energy state is defined by the real solution of the algebraic equation (equation (58)) plus the expression given in equation (59) for the radial mode $n=1$, instead of $n=0$. This effect arises due to the presence of Cornell-type potential in the system. Note that it is necessary physically that the lowest energy state is $n=1$ and not $n=0$; otherwise, the opposite would imply that $c_{1}=0$ which is not possible.
The corresponding radial wave function for $n=1$ is given by

$$
\psi_{1, l}(r)=r{\sqrt{\eta_{c}^{2}-e^{2} \xi_{c}^{2}}}^{-(1 / 2)}\left(\begin{array}{c}
r+(b /(3 / 2)) \\
\tilde{\omega}_{1, l}
\end{array}\right) r\left(c_{0}+c_{1} r\right),
$$

where

$c_{1}=\frac{1}{\sqrt{\tilde{\omega}_{1, l}}}\left[\frac{2\left(e \xi_{c} E_{1, l}+m \eta_{c}\right)}{1+2 \sqrt{\eta_{c}^{2}-e^{2} \xi_{c}^{2}}}+\frac{2\left(m \eta_{L}+\omega_{1, l}\left(l-2 e \Omega \xi_{c}\right)\right)}{2 \tilde{\omega}_{1, l}}\right] c_{0}$.

\section{Conclusions}

The relativistic quantum system of scalar and spinhalf particles in Gödel-type space-times was investigated by several authors (e.g., [7-9, 11-14, 44, 59]). They demonstrated that the energy eigenvalues of the relativistic quantum system get modified and depend on the global parameters characterizing the space-times.

In this work, we have investigated the influence of vorticity parameter on the relativistic energy eigenvalues of a relativistic scalar particle in Gödel-type space-time subject to a homogeneous magnetic field with potentials. We have derived the radial wave equation of the Klein-Gordon equation in a class of flat Gödel-type space-time in the presence of external fields with or without potentials by choosing a suitable ansatz of the wave function. In Section 2.1, we have introduced Cornell-type scalar and Coulomb-type vector potentials into the considered relativistic system and obtained the energy eigenvalue (equation (23)) and corresponding eigenfunctions (equation (24)). We have seen that the presence of a uniform magnetic field and potential parameters modifies the energy spectrum in comparison to those results obtained in [12]. By imposing the additional recurrence condition $c_{n+1}=0$, we have obtained the ground state energy levels (equation (26)) and wave functions (equations (27) and (28)) for $n=1$. In Section 2.2, we have considered zero potential into the relativistic system and solved the radial wave equation of the Klein-Gordon equation in the presence of an external field. We obtained the energy eigenvalues (equation (35)) and compared them with the results obtained in [12]. We have seen that the relativistic energy eigenvalues (equation (35)) get modified in comparison to those in [12] due to the presence of a homogeneous magnetic field. In Section 3, we have solved the Klein-Gordon equation of the Klein-Gordon oscillator in Gödel-type space-time subjected to a homogeneous magnetic field in the presence of Cornell-type scalar and Coulomb-type vector potentials. We have obtained the energy eigenvalue (equation (56)) and corresponding eigenfunctions (equation (57)). We have seen that the presence of a uniform magnetic field and potential parameters modifies the energy spectrum in comparison to those in [12]. By imposing the additional recurrence condition $c_{n+1}=0$, we have obtained the ground state energy 
levels (equation (59)) and wave function (equation (60)) for $n=1$ and others are in the same way.

So, in this paper, we have some results which are in addition to the previous results obtained in $[7-9,11-13,44,51$, 59] which may present interesting effects. This is the fundamental subject in physics, and the connection between these theories (quantum mechanics and gravitation) is not well understood.

\section{Data Availability}

No data has been used to prepare this paper.

\section{Conflicts of Interest}

The author declares that there is no conflict of interest regarding the publication of this paper.

\section{References}

[1] K. Gödel, "An example of a new type of cosmological solutions of Einstein's field equations of gravitation," Reviews of Modern Physics, vol. 21, no. 3, pp. 447-450, 1949.

[2] M. J. Reboucas and J. Tiomno, "Homogeneity of Riemannian space-times of Gödel type,” Physical Review D, vol. 28, no. 6, pp. 1251-1264, 1983.

[3] M. Reboucas, J. Aman, and A. F. F. Teixeira, "A note on Gödeltype spacetimes," Journal of Mathematical Physics, vol. 27, article 1370, 1985.

[4] M. O. Galvao, M. Reboucas, A. F. F. Teixeira, and W. M. Silva, "Notes on a class of homogeneous space-times," Journal of Mathematical Physics, vol. 29, p. 1127, 1988.

[5] F. Ahmed, "The Dirac equation in a class of topologically trivial flat Gödel-type space-time backgrounds," European Physical Journal C: Particles and Fields, vol. 79, no. 6, p. 534, 2019.

[6] B. D. Figueiredo, I. D. Soares, and J. Tiomno, "Gravitational coupling of Klein-Gordon and Dirac particles to matter vorticity and spacetime torsion," Classical and Quantum Gravity, vol. 9, no. 6, pp. 1593-1617, 1992.

[7] N. Drukker, B. Fiol, and J. Simon, "Gödel-type universe and Landau problem," Journal of Cosmology and Astroparticle Physic, vol. 410, p. 12, 2004.

[8] S. Das and J. Gegenberg, "Gravitational non-commutativity and Gödel-like spacetimes," General Relativity and Gravitation, vol. 40, no. 10, pp. 2115-2129, 2008.

[9] J. Carvalho, A. M. M. Carvalho, and C. Furtado, "Quantum influence of topological defects in Gödel-type space-times," The European Physical Journal C, vol. 74, no. 6, article 2935, 2014.

[10] J. Carvalho, A. M. M. Carvalho, E. Cavalcante, and C. Furtado, "Klein-Gordon oscillator in Kaluza-Klein theory," The European Physical Journal C, vol. 76, no. 7, p. 365, 2016.

[11] Z. Wang, Z. Long, C. Long, and M. Wu, "Relativistic quantum dynamics of a spinless particle in the Som-Raychaudhuri space-time," The European Physical Journal Plus, vol. 130, no. 3, p. 36, 2015.

[12] F. Ahmed, "The energy-momentum distributions and relativistic quantum effects on scalar and spin-half particles in a Gödel-type space-time," European Physical Journal C: Particles and Fields, vol. 78, no. 7, p. 598, 2018.
[13] F. Ahmed and H. Hassanabadi, "Spin-0 system of DKP equation in the background of a flat class of Gödel-type spacetime," Modern Physics Letters A, vol. 35, no. 7, p. 2050031, 2020.

[14] R. L. L. Vitória, C. Furtado, and K. Bakke, "Linear confinement of a scalar particle in a Gödel-type spacetime," The European Physical Journal C, vol. 78, no. 1, p. 44, 2018.

[15] F. Ahmed, "Comment on "Linear confinement of a scalar particle in a Gödel-type space-time [Eur. Phys. J. C (2018) 78: 44]"," European Physical Journal C: Particles and Fields, vol. 79, no. 8, p. 682, 2019.

[16] M. Eshghi and M. Hamzavi, "Yukawa-like confinement potential of a scalar particle in a Gödel-type spacetime with any $l$," European Physical Journal C: Particles and Fields, vol. 78, no. 6 , p. 522, 2018.

[17] E. O. Silva, "Ground state of a bosonic massive charged particle in the presence of external fields in a Gödel-type spacetime," The European Physical Journal Plus, vol. 133, no. 12, p. 530, 2018.

[18] F. Ahmed, "Comment on "Ground state of a bosonic massive charged particle in the presence of external fields in a Gödeltype space-time [Eur. Phys. J. Plus (2018) 133: 530]"," The European Physical Journal Plus, vol. 135, no. 2, p. 174, 2020.

[19] F. Ahmed, "Relativistic quantum dynamics of spin-0 massive charged particle in the presence of external fields in 4D curved space-time with a cosmic string," The European Physical Journal Plus, vol. 135, no. 1, p. 108, 2020.

[20] F. Ahmed, "Scalar quantum particle in $(1+2)$-dimensions Gurses space-time and the energy-momentum distributions," Annals of Physics, vol. 401, no. 193, pp. 193-201, 2019.

[21] F. Ahmed, "The Klein-Gordon oscillator in (1+2)-dimensions Gurses space-time backgrounds," Annals of Physics, vol. 404, no. 1, pp. 1-9, 2019.

[22] F. Ahmed, "The generalized Klein-Gordon oscillator with Coulomb-type potential in (1+2)-dimensions Gürses spacetime," General Relativity and Gravitation, vol. 51, no. 5, p. $69,2019$.

[23] F. Ahmed, "Relativistic quantum motion of spin-0 particles with a Cornell-type potential in $(1+2)$-dimensional Gürses spacetime backgrounds," Modern Physics Letters A, vol. 34, no. 38, p. 1950314, 2019.

[24] F. Ahmed, "Linear confinement of spin-0 relativistic quantum particles in (1+2)-dimensions Gürses space-time," Annals of Physics, vol. 411, no. 167941, p. 167941, 2019.

[25] F. Ahmed, "Quantum effects on spin-0 massive charged particles with Coulomb-type potentials in $(1+2)$-dimensions Gürses space-time," General Relativity and Gravitation, vol. 51, no. 10, p. 129, 2019.

[26] G. Q. Garcia, J. R. S. Oliveira, K. Bakke, and C. Furtado, "Fermions in Gödel-type background space-times with torsion and the Landau quantization," The European Physical Journal Plus, vol. 132, no. 3, p. 123, 2017.

[27] G. Q. Garcia, J. R. . S. Oliveira, and C. Furtado, "Weyl fermions in a family of Gödel-type geometries with a topological defect," International Journal of Modern Physics D, vol. 27, no. 3, article $1850027,2018$.

[28] S. G. Fernandes, G. . A. Marques, and V. B. Bezerra, "Scalar solutions in spacetimes containing a cosmic string," Classical and Quantum Gravity, vol. 23, no. 23, pp. 7063-7073, 2006.

[29] A. Havare and T. Yetkin, "Exact solution of the photon equation in stationary G\$ouml\$del-type and G\$ouml\$del spacetimes," 
Classical and Quantum Gravity, vol. 19, no. 11, pp. 2783-2791, 2002.

[30] L. C. N. Santos and C. C. Barros Jr., "Dirac equation and the Melvin metric," European Physical Journal C: Particles and Fields, vol. 76, no. 10, p. 560, 2016.

[31] M. de Montigny, S. Zare, and H. Hassanabadi, "Fermi field and Dirac oscillator in a Som-Raychaudhuri space-time," General Relativity and Gravitation, vol. 50, no. 5, p. 47, 2018.

[32] P. Sedaghatnia, H. Hassanabadi, and F. Ahmed, "Dirac fermions in Som-Raychaudhuri space-time with scalar and vector potential and the energy momentum distributions," European Physical Journal C: Particles and Fields, vol. 79, no. 6, p. 541, 2019.

[33] F. Ahmed, "The Dirac equation in $(1+2)$-dimensional Gürses space-time backgrounds," The European Physical Journal Plus, vol. 134, no. 10, p. 518, 2019.

[34] Q. Wen-Chao, "Bound states of the Klein-Gordon and Dirac equations for potential $V(r)=A \bar{r}^{2}-B \bar{r}^{1}$," Chinese Physics, vol. 12, no. 10, pp. 1054-1057, 2003.

[35] H. Motavalli and A. R. Akbarieh, "Klein-Gordon equation for the Coulomb potential in noncommutative space," Modern Physics Letters A, vol. 25, no. 29, pp. 2523-2528, 2011.

[36] F. Yasuk, A. Durmus, and I. Boztosun, "Exact analytical solution to the relativistic Klein-Gordon equation with noncentral equal scalar and vector potentials," Journal of Mathematical Physics, vol. 47, no. 8, article 082302, 2006.

[37] A. L. C. de Oliveira and E. R. Bezerra de Mello, "Exact solutions of the Klein-Gordon equation in the presence of a dyon, magnetic flux and scalar potential in the spacetime of gravitational defects," Classical and Quantum Gravity, vol. 23, no. 17, pp. 5249-5263, 2006.

[38] H. Asada and T. Futamase, "Propagation of gravitational waves from slow motion sources in a Coulomb-type potential," Physical Review D, vol. 56, no. 10, pp. R6062-R6066, 1997.

[39] C. L. Critchfield, "Scalar potentials in the Dirac equation," Journal of Mathematical Physics, vol. 17, no. 2, pp. 261-266, 1976.

[40] H. W. Crater, J.-H. Yoon, and C. Y. Wong, "Singularity structures in Coulomb-type potentials in two-body Dirac equations of constraint dynamics," Physical Review D, vol. 79, no. 3, article 034011, 2009.

[41] V. R. Khalilov, "Relativistic Aharonov-Bohm effect in the presence of planar Coulomb potentials," Physical Review A, vol. 71, no. 1, article $012105,2005$.

[42] E. R. Figueiredo Medeiros and E. R. Bezerra de Mello, "Relativistic quantum dynamics of a charged particle in cosmic string spacetime in the presence of magnetic field and scalar potential," European Physical Journal C: Particles and Fields, vol. 72, no. 6, p. 2051, 2012.

[43] K. Bakke and C. Furtado, "On the Klein-Gordon oscillator subject to a Coulomb-type potential," Ann. Phys., vol. 355, pp. 48-54, 2015.

[44] F. Ahmed, "Linear confinement of a scalar and spin-0 particle in a topologically trivial flat Gödel-type space-time," European Physical Journal C: Particles and Fields, vol. 79, no. 2, p. 104, 2019.

[45] L. C. N. Santosa and C. C. Barros Jr., "Relativistic quantum motion of spin-0 particles under the influence of noninertial effects in the cosmic string space-time," European Physical Journal C: Particles and Fields, vol. 78, no. 1, p. 13, 2018.
[46] R. L. L. Vitoria, C. Furtado, and K. Bakke, "On a relativistic particle and a relativistic position-dependent mass particle subject to the Klein-Gordon oscillator and the Coulomb potential," Ann. Phys., vol. 370, pp. 128-136, 2016.

[47] M. Hosseinpour, H. Hassanabadi, and M. de Montigny, "Klein-Gordon field in spinning cosmic-string space-time with the Cornell potential," International Journal of Geometric Methods in Modern Physics, vol. 15, no. 10, article 1850165, 2018.

[48] H. Sobhani, H. Hassanabdi, and W. S. Chung, "A survey on the Klein-Gordon equation in the Gödel-type space-times," International Journal of Geometric Methods in Modern Physics, vol. 15, no. 3, article 1850037, 2018.

[49] G. B. Arfken and H. J. Weber, Mathematical Methods for Physicists, Academic Press, Elsevier, 2005.

[50] F. Ahmed, "A type D non-vacuum spacetime with causality violating curves, and its physical interpretation," Communications in Theoretical Physics, vol. 68, no. 6, p. 735, 2017.

[51] F. Ahmed, "Relativistic quantum dynamics of spin-0 system of the DKP oscillator in a Gödel-type space-time," Communications in Theoretical Physics, vol. 72, no. 2, article 025103, 2020.

[52] D. H. Perkins, An Introduction to High Energy Physics, Cambridge University Press, Cambridge, 2000.

[53] M. K. Bahar and F. Yasuk, "Exact Solutions of the MassDependent Klein-Gordon Equation with the Vector QuarkAntiquark Interaction and Harmonic Oscillator Potential," Advances in High Energy Physics, vol. 2013, Article ID 814985, 6 pages, 2013.

[54] C. Alexandrou, P. de Forcrand, and O. Jahn, "The ground state of three quarks," Nuclear Physics B - Proceedings Supplements, vol. 119, no. 667, pp. 667-669, 2003.

[55] J. D. Garret and M. S. Lozano, "Spectroscopy, leptonic decays and the nature of heavy quarkonia," Physics Letters B, vol. 669, no. 1, pp. 52-57, 2008.

[56] E. Eichten, K. Gottfried, T. Kinoshita, K. D. Lane, and T. M. Yan, "Charmonium: comparison with experiment," Physical Review D, vol. 21, no. 1, pp. 203-233, 1980.

[57] E. Eichten, K. Gottfried, T. Kinoshita, K. D. Lane, and T. Yan, "Erratum: charmonium: the model," Physical Review D, vol. 21, no. 1, p. 313, 1980.

[58] M. S. Cunha, C. R. Muniz, H. R. Christiansen, and V. B. Bezerra, "Relativistic Landau levels in the rotating cosmic string spacetime," European Physical Journal C: Particles and Fields, vol. 76, no. 9, p. 512, 2016.

[59] R. L. L. Vitória and K. Bakke, "Relativistic quantum effects of confining potentials on the Klein-Gordon Oscillator," The European Physical Journal Plus, vol. 131, no. 2, p. 36, 2016.

[60] R. L. L. Vitória and H. Belich, "A central potential with a massive scalar field in a Lorentz symmetry violation environment," Advances in High Energy Physics, vol. 2019, Article ID 1248393, 13 pages, 2019.

[61] R. L. L. Vitória and K. Bakke, "On the interaction of the scalar field with a Coulomb-type potential in a spacetime with a screw dislocation and the Aharonov-Bohm effect for bound states," The European Physical Journal Plus, vol. 133, no. 11, p. 490, 2018.

[62] R. L. L. Vitória and H. Belich, "Effects of a linear central potential induced by the Lorentz symmetry violation on the KleinGordon oscillator," The European Physical Journal C, vol. 78, no. 12, p. 999, 2018. 
[63] E. V. B. Leite, H. Belich, and R. L. L. Vitória, "Effects of the Cornell-type potential on a position-dependent mass system in Kaluza-Klein theory," Advances in High Energy Physics, vol. 2019, Article ID 6740360, 7 pages, 2019.

[64] L. F. Deng, C.-Y. Long, Z.-W. Long, and T. Xu, "Generalized Dirac Oscillator in Cosmic String Space-Time," Advances in High Energy Physics, vol. 2018, Article ID 2741694, 10 pages, 2018.

[65] A. Ronveaux, Heuns Differential Equations, Oxford University Press, Oxford, 1995.

[66] S. Y. Slavyanov and W. Lay, Special Functions: A Unified Theory Based in Singularities, Oxford University Press, New York, NY, USA, 2000.

[67] A. Vercin, "Two anyons in a static uniform magnetic field. Exact solution," Physics Letters B, vol. 260, no. 1-2, pp. 120124, 1991.

[68] J. Myrheim, E. Halvorsen, and A. Verçin, "Two anyons with Coulomb interaction in a magnetic field," Physics Letters B, vol. 278, no. 1-2, pp. 171-174, 1992.

[69] S. Bruce and P. Minning, "The Klein-Godron oscillator," II Nuovo Cimento A, vol. 106, p. 711, 1993.

[70] V. V. Dvoeglazov, "Comment on "The Klein-Gordon oscillator by S. Bruce and P. Minning"," II Nuovo Cimento A, vol. 107, article 1413, 1994.

[71] B. Mirza and M. Mohadesi, "The Klein-Gordon and the Dirac oscillators in a noncommutative space," Communications in Theoretical Physics, vol. 42, no. 5, pp. 664-668, 2004. 